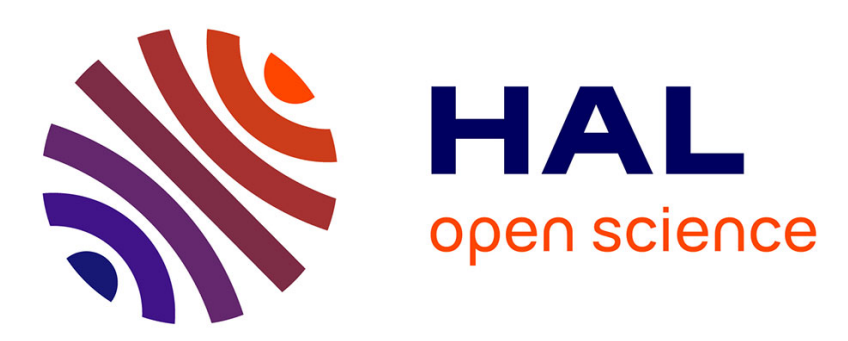

\title{
A torsion test for the study of the large deformation recovery of shape memory polymers
}

Julie Diani, Carole Fredy, Pierre Gilormini, Yannick Merckel, Gilles Regnier, Ingrid Rousseau

\section{- To cite this version:}

Julie Diani, Carole Fredy, Pierre Gilormini, Yannick Merckel, Gilles Regnier, et al.. A torsion test for the study of the large deformation recovery of shape memory polymers. Polymer Testing, 2011, 30 (3), pp.335-341. 10.1016/j.polymertesting.2011.01.008 . hal-00755438

\section{HAL Id: hal-00755438 \\ https://hal.science/hal-00755438}

Submitted on 21 Nov 2012

HAL is a multi-disciplinary open access archive for the deposit and dissemination of scientific research documents, whether they are published or not. The documents may come from teaching and research institutions in France or abroad, or from public or private research centers.
L'archive ouverte pluridisciplinaire HAL, est destinée au dépôt et à la diffusion de documents scientifiques de niveau recherche, publiés ou non, émanant des établissements d'enseignement et de recherche français ou étrangers, des laboratoires publics ou privés. 


\title{
A torsion test for the study of the large deformation recovery of shape memory polymers
}

\author{
Julie Diani ${ }^{\mathrm{a}}$, Carole Frédy ${ }^{\mathrm{a}}$, Pierre Gilormini ${ }^{\mathrm{a}}$, Yannick Merckel ${ }^{\mathrm{b}}$, Gilles Régnier ${ }^{\mathrm{a}}$, Ingrid \\ Rousseau $^{\mathrm{c}}$ \\ a PIMM, CNRS, Arts et Métiers ParisTech, 151 bd de l'Hôpital, 75013 Paris, FRANCE. \\ ${ }^{\mathrm{b}}$ LML, Ecole Centrale de Lille, bd Paul Langevin, 59650 Villeneuve d'Ascq, FRANCE. \\ ${ }^{\mathrm{c}}$ General Motors Company, Research \& Development Center, 30500 Mound Rd, \\ Warren, MI 48090-9055, USA.
}

Keywords: Shape memory polymer, Strain recovery, Large deformation, Torsion test, Thermoelasticity, Image analysis

\begin{abstract}
:
A torsion device was designed and built for testing the shape fixity and shape recovery of shape memory polymers at large deformation. A simple thermal chamber was used to regulate the thermal environment during testing and a CCD camera was used for recording the deformation. Such a torsion testing system provided a quantitative estimate of the kinematics and kinetics of shape recovery for samples submitted to large deformations at moderate strains that are more likely expected in actual shape memory applications. In addition, such measurements are complementary to those obtained from large strain uniaxial tension tests usually run for during traditional shape memory effect characterization. As a result, the torsional shape memory testing device and testing method described is expected to contribute building complementary data for the thermomechanical modeling of shape memory polymers.
\end{abstract}

\section{Introduction}

Shape memory polymers (SMPs) are chemically or physically crosslinked networks, which have the capability to retain a temporary shape and recover their original, permanent shape in a controlled manner using an external stimulus. A deformation at high temperature, above a material-specific transformation temperature, results in forming the temporary shape, which is thereafter set by cooling to below the SMP's transformation temperature. Indeed, 
above such temperature, polymer networks may undergo relatively large elastic deformations due to the thermal activation of the molecular mobility. When deformed then cooled below the transformation temperature (i.e., most commonly a glass transition or melting transition temperature), the chain mobility declines and the molecular chains freeze, thereby adopting the conformations set by the deformation. Furthermore, the restrained molecular mobility enables the SMP to remain in the temporary shape even after the deforming stresses are released. It is only by applying an external stimulation, often simply heat, that the recovery of the SMP's original, permanent shape is triggered. The shape recovery is due to the release of the elastic strain (entropic in nature) that was input and stored during the deformation and the shape fixing stages, respectively. The steps involved during a thermo-mechanical cycle representative of the shape fixing and the shape recovery for a shape memory polymer are illustrated in Fig. 1.

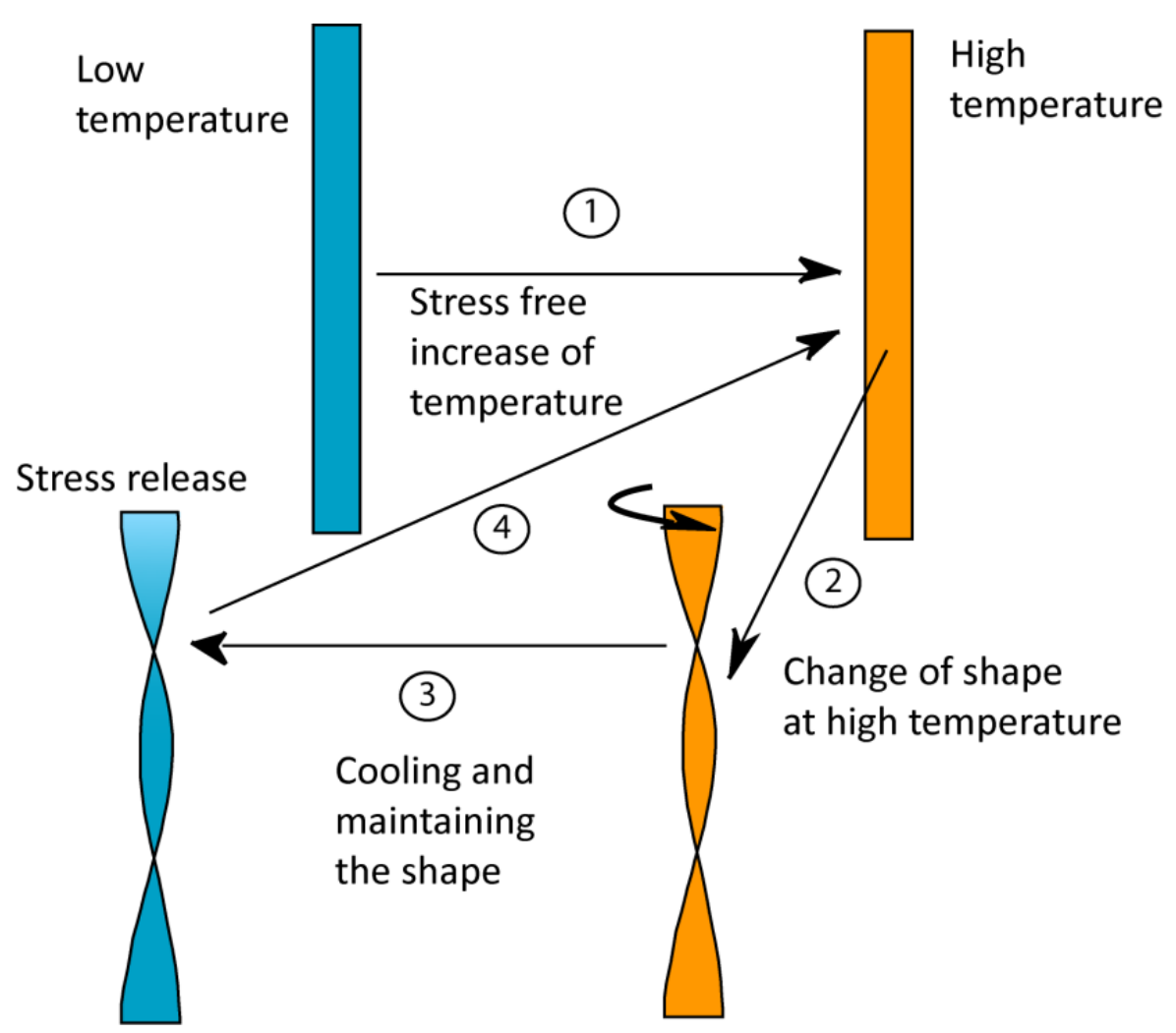

Fig. 1. Illustration of a shape storage/shape recovery thermomechanical cycle of an SMP.

Recently, scientists have been developing SMPs as an alternative to using shape memory alloys. Applications targeted include intelligent biomedical devices and industrial applications due to the many advantageous features that polymers offer over metal alloys [1]. Indeed, SMPs are capable of very large deformations, which is essential for applications where storage space is critical like in aerospace applications [2,3] . In addition, a large 
number of polymers are food and/or drug compatible making these materials good candidates for biomedical applications $[4,5]$. Also, the fact that SMPs' transformation temperature can be easily tailored through molecular composition and structure [6,7] is a great advantage over their metal alloys counterparts. Finally, they are stable over a wide range of temperature, from below $-100{ }^{\circ} \mathrm{C}$ to above $+200{ }^{\circ} \mathrm{C}$, they are light, generally inexpensive, and easier to process compared to shape memory alloys [1].

The development of new technical applications requires improvements in the characterization and the modeling of the thermomechanical behavior of these materials. The shape memory properties are characterized by many quantities such as the activation temperature of the shape recovery, the shape fixity, which designates the extent of shape retention between the applied temporary shape at high temperature and the memorized temporary shape at low temperatures after the deforming stresses were released, the shape recovery, which quantifies the shape recovery performance after a full SM thermomechanical cycle relative to the initial, permanent shape to be recovered, the kinematics and kinetics of recovery, amongst others. Usually, the kinematics of the shape recovery is either measured quantitatively in uniaxial tension [8-11] or uniaxial compression [12-14] or discussed qualitatively for various types of large deformation loadings [15], including bending [16-18] and rod to spiral twisting [7,19]. Hence, the mechanical models developed to date are mostly fit and validated for uniaxial tension recovery tests where large deformation loading conditions would be more representative of the applications. For this reason, a torsion device allows the quantitative measure of the kinematics and kinetics of the shape recovery of polymer materials when submitted to large deformations and moderate strains. This device provided an easy way to gather unprecedented experimental data for the characterization of the shape memory property of polymers submitted to large deformations at moderate strains. The data are complementary to those obtained from large uniaxial tension strains reported in the literature.

Here, the original torsion device is presented along with details about the shape memory testing protocol as well as the data collection and analysis methods. Advantageously, the test environment did not require elaborate equipment; a simple thermal chamber, a CCD camera and personal computer were used. Tests were run using well-known, glass transitionresponsive, thermoset and thermoplastic shape memory polymers and demonstrated the universal character of the device proposed with regards to testing, quantifying and comparing the shape memory properties of structurally and molecularly different SMPs. The kinematics and kinetics of the torsion angle recovery as a function of temperature, heating rate, maximal recovery temperature and extent of exposure at the maximal recovery temperature, state of deformation, and material structure are presented and briefly discussed. 


\section{Experimental}

\subsection{Torsion recovery test}

\subsubsection{Torsion test device}

A mechanical device was designed to apply torsion loadings to rectangular specimens and further monitor their torsion angle recovery when heated. Torsion loading conditions induce large isochoric deformations, and are complementary to large strain uniaxial tension conditions. Also, torsions involve large deformations at moderate strains that are advantageous for materials with limited elastic strain regions, like in thermoplastic elastomers.

The device is shown in Fig. 2. A slender rectangular sample (100 mm long, $10 \mathrm{~mm}$ wide and 1 to $4 \mathrm{~mm}$ thick) is fixed at its bottom end to the bottom of the device (Part B). The top end of the sample is bound to the top part of the device (Part A) by a prismatic joint. Part A is mobile. It can be rotated around the sample's longitudinal axis thereby leading to a torsion deformation of the rectangular specimen. Its angular position can be secured by a locating pin. Part A can also be removed from the main body.

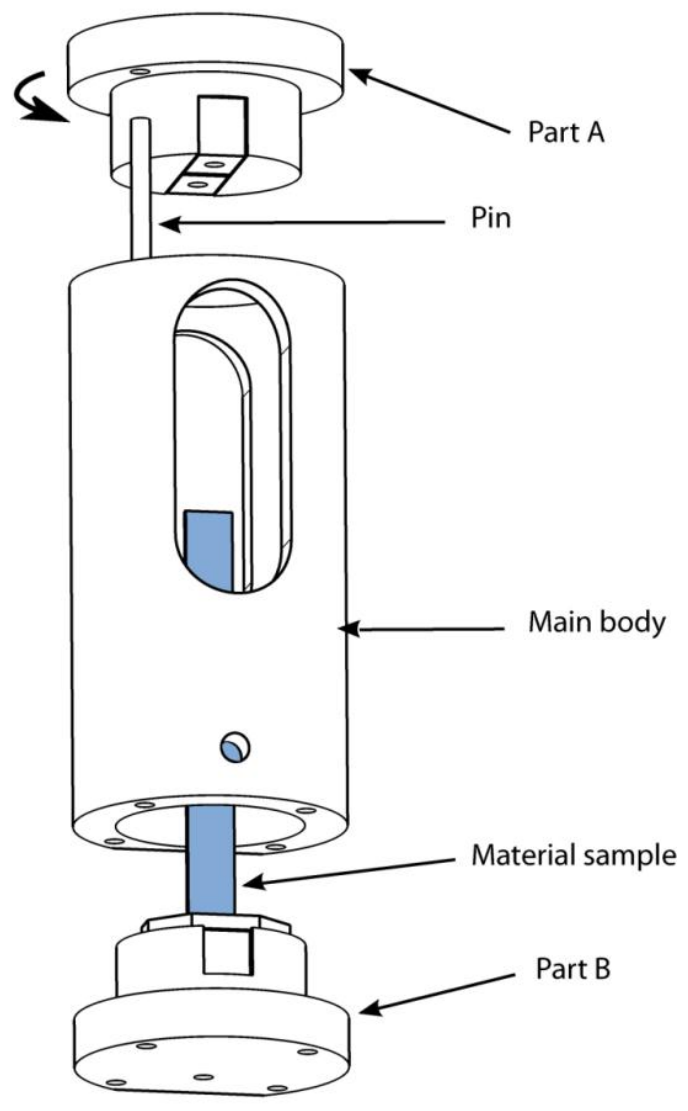

Fig. 2. Torsion device. 


\subsubsection{Experimental protocol}

\subsubsection{Setting of a Temporary Shape}

After being cut to rectangular dimensions of $100 \times 10 \times 1 \mathrm{~mm}^{3}$, the specimen is set in the torsion device as detailed above. The torsion device is then introduced inside a thermal chamber for heating. A standard XU125 thermal chamber from MTC was used. Once heated above its glass transition, the material sample is submitted to the desired torsion deformation, which is characterized by the rotation angle imparted to its top end (Part A). The deformation is kept constant by inserting the locating pin in position. The material is then cooled down to a temperature below its glass transition. Once cooled, the stresses are released by removing Part A from the main body.

\subsubsection{Monitoring of the Torsion Angle Recovery}

Once the desired temporary shape has been set, the next step consists in setting the torsion device in such a way that recovery can be monitored and recorded. For this purpose, the motion of the free end of the specimen is captured by a CCD Dalsa Genie M1024 camera equipped with a Nikon Sigma DG lens. During this step the torsion device is installed upside down within the thermal chamber to prevent any unwanted bending or folding of the specimen that may arise from gravity forces. Therefore, the video capture of sample's motion is enabled through its reflection in a 45 o tilted mirror that sits at the below the inverted torsion device (Fig. 3). The camera features a 1024 x 876 pixels resolution with a pixel size of 4.65 microns. The images are captured using CamExpert software from Dalsa and CamStudio free software from RenderSoft. The temperature is monitored by using a standard thermocouple introduced in a strain-free control sample positioned in close proximity to the sample being tested. 


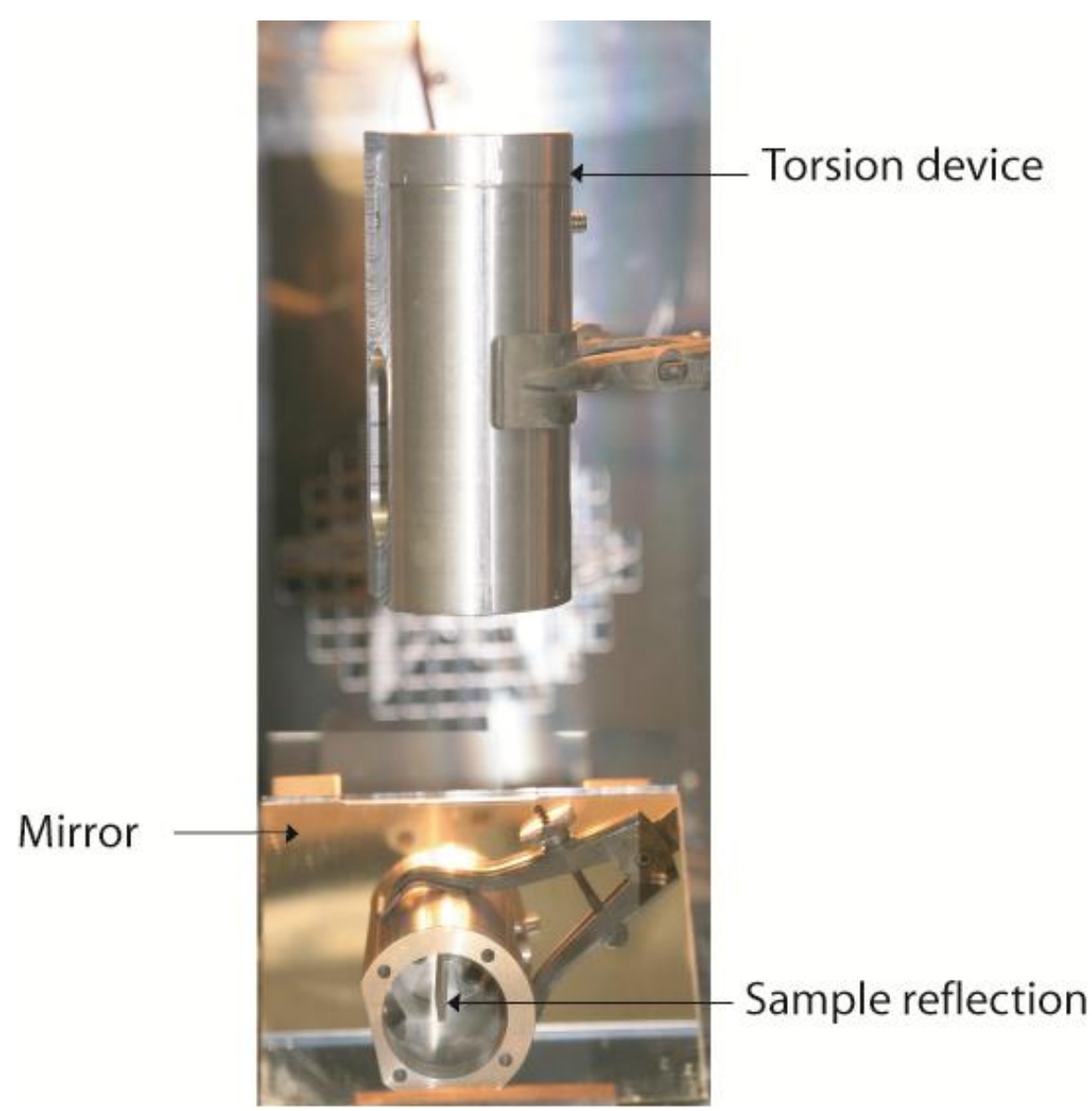

Fig. 3. Torsion device set upside down in the thermal chamber. The angular motion of the sample free end is monitored through video capture of its reflection from a $45^{\circ}$ tilted mirror.

\subsubsection{Deformation measurements}

During angle recovery, the motion of the sample free end is captured as an AVI file format, from which a periodic sequence of images can be extracted. In order to monitor the angle of deformation as a function of time and temperature the edge of the sample was pinpointed with two painted markers (Fig. 4), which trajectories during shape recovery were obtained using a particle tracker plug-in developed for the imageJ free software [20] in which the point detection and tracking algorithm was initially implemented for monitoring cells motions. It is fully described in [21]. The initial and the final images provide access to the initial elastic recovery and the permanent set (unrecovered) deformation, respectively. 


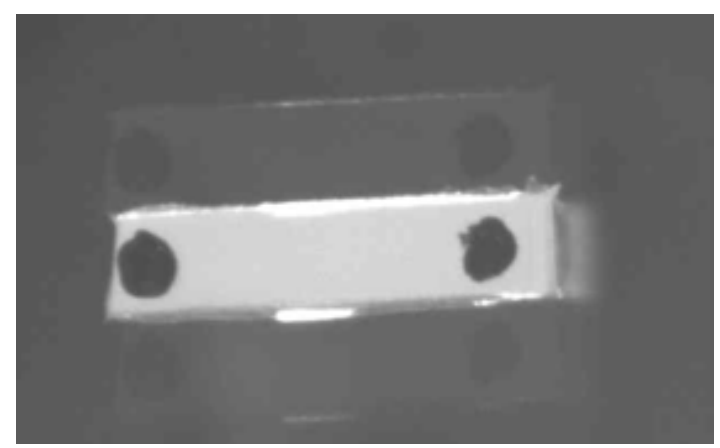

Fig. 4. Painted markers on the sample free end for detection and tracking of the angle motion during shape recovery.

\subsection{Materials}

Two materials were selected, a thermoplastic polyether block amid elastomer (Pebax® 7233) kindly supplied by Arkema, and a glassy amorphous epoxy network developed at General Motors. The epoxy network is composed ofa DOW D.E.R. 383 epoxy resin crosslinked with a mixture of Jeffamine D-148 and Jeffamine ED-2003 from Huntsman Corp. The glass transition temperatures of both materials were determined by dynamic mechanical analysis (DMA) in a tensile mode using a Q800 DMA from TA Instruments. All tests were performed at a frequency of $1 \mathrm{~Hz}$ and a heating rate of $2{ }^{\circ} \mathrm{C} / \mathrm{min}$. The epoxy and the Pebax were submitted to a strain of $0.2 \%$ and $0.1 \%$ respectively (the Pebax being stiffer). Fig. 5 shows the DMA curves. The epoxy network exhibited a sharp glass-rubber transition which onsets just above $40{ }^{\circ} \mathrm{C}$. Its glass transition, determined by the peak of the $\tan \delta$ curve, arises at $57{ }^{\circ} \mathrm{C}$. The rubbery region is clearly identified by a storage modulus plateau at high temperatures. The Pebax glass-rubber transition starts around $20{ }^{\circ} \mathrm{C}$ and extends over a wide temperature window. Its glass transition occurs at $35{ }^{\circ} \mathrm{C}$. Unlike the covalently crosslinked epoxy, the rubbery region of the physically crosslinked Pebax is characterized by a slowly decreasing storage modulus with increasing temperature. These materials were in fact selected due to their contrasting temperature-dependent behaviors. 


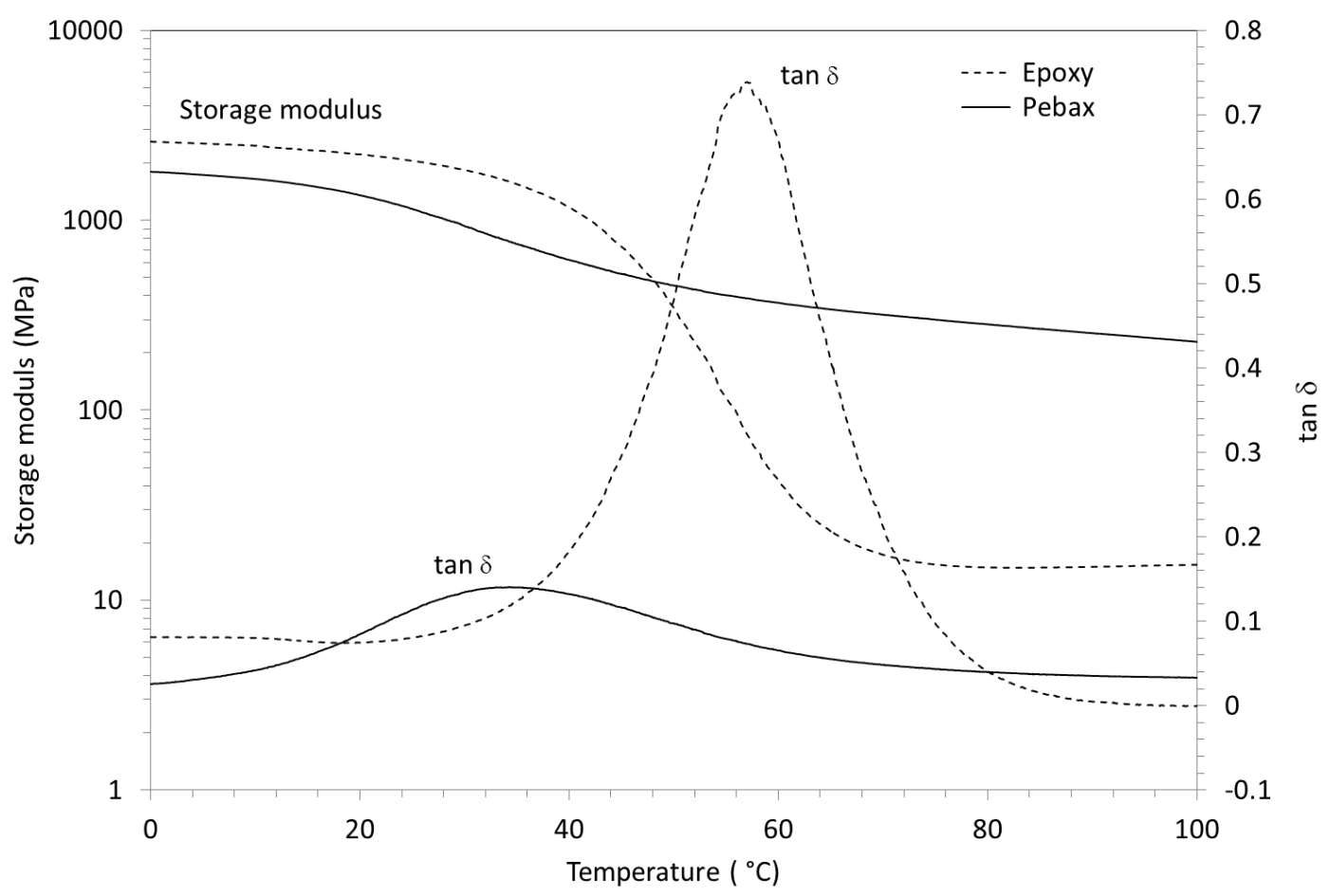

Fig. 5. DMA curves for the epoxy and the Pebax shape memory polymers.

\subsection{Test conditions}

Epoxy and Pebax samples were cut and set into the torsion device, then heated above their respective glass transition temperature (i.e., to $80^{\circ} \mathrm{C}$ for the epoxy and to $150{ }^{\circ} \mathrm{C}$ for the Pebax) and maintained at temperature for approximately ten minutes. Then the samples were deformed in torsion, their tops being twisted at 180,270 , or $360^{\circ}$ angles. While the torsion angle was maintained, the sample was cooled slowly to room temperature. At this temperature, below the glass transition, the stresses were released by removing the top part of the torsion device (Part A in Fig. 2). An angle recovery of several degrees may be observed upon releasing the stresses. It is linked to the shape fixity characteristic of the shape memory polymer. Finally, the torsion device containing the sample deformed in its temporary, twisted shape was placed inside the thermal chamber and heated. The heating procedure was characterized by a maximal temperature and a temperature ramp which was varied from 0.9 to $3.6{ }^{\circ} \mathrm{C} / \mathrm{min}$. While the sample was heated, the temperature and the motion of the sample's free end were recorded as functions of time. This allowed the quantification of the shape recovery as a function of time and temperature.

\section{Results}

3.1. Quantitative measurements of the shape fixity and the shape recovery at large deformation 
In the following, torsional shape recovery results obtained using the torsion device are discussed.

First, the test reproducibility was demonstrated by applying 360-degree torsion (i.e., one full twist) on the epoxy network, and by measuring the elastic recovery at low temperature and the shape recovery during a $0.9{ }^{\circ} \mathrm{C} / \mathrm{min}$ heating ramp. The material was heated to $80{ }^{\circ} \mathrm{C}$ at which point it was submitted to a 360-degree twist, then cooled to room temperature while maintaining the deformation. At low temperatures the stresses were released and the instantaneous elastic angular recovery was measured to be about $18^{\circ}$. This provided an estimate of the material shape fixity, $R_{f}$, which characterizes the difference between the deformation measured after applying a deformation at high temperatures, cooling and unloading, and the applied deformation at high temperatures. In torsion, $R_{f}$ may be defined by:

$$
R_{f}=\frac{\text { Angle of torsion after unloading }}{\text { Angle of torsion applied }}
$$

Therefore, $R_{f}$ of the epoxy network tested here is about $95 \%$ for a $360^{\circ}$ deformation angle of a sample of dimension $100 \times 10 \times 1 \mathrm{~mm}^{3}$.

The angular recovery of the samples was monitored while applying a heating ramp of 0.9 ${ }^{\circ} \mathrm{C} / \mathrm{min}$. Fig. 6 shows the angular recovery as a function of temperature for two fresh samples. One notes the good reproducibility of the test. The shape recovery of the epoxy networks appears complete (i.e., $~ 100 \%$ ). Fig. 6 shows a quantitative measurement of the kinematics of recovery. Deriving the recovery kinematics gives access to the kinetics of recovery, which is presented in Fig. 7. When the temperature rises above that of the onset of the glass transition, $40{ }^{\circ} \mathrm{C}$, the shape recovery proceeds quickly to reach a maximum before slowing down. 


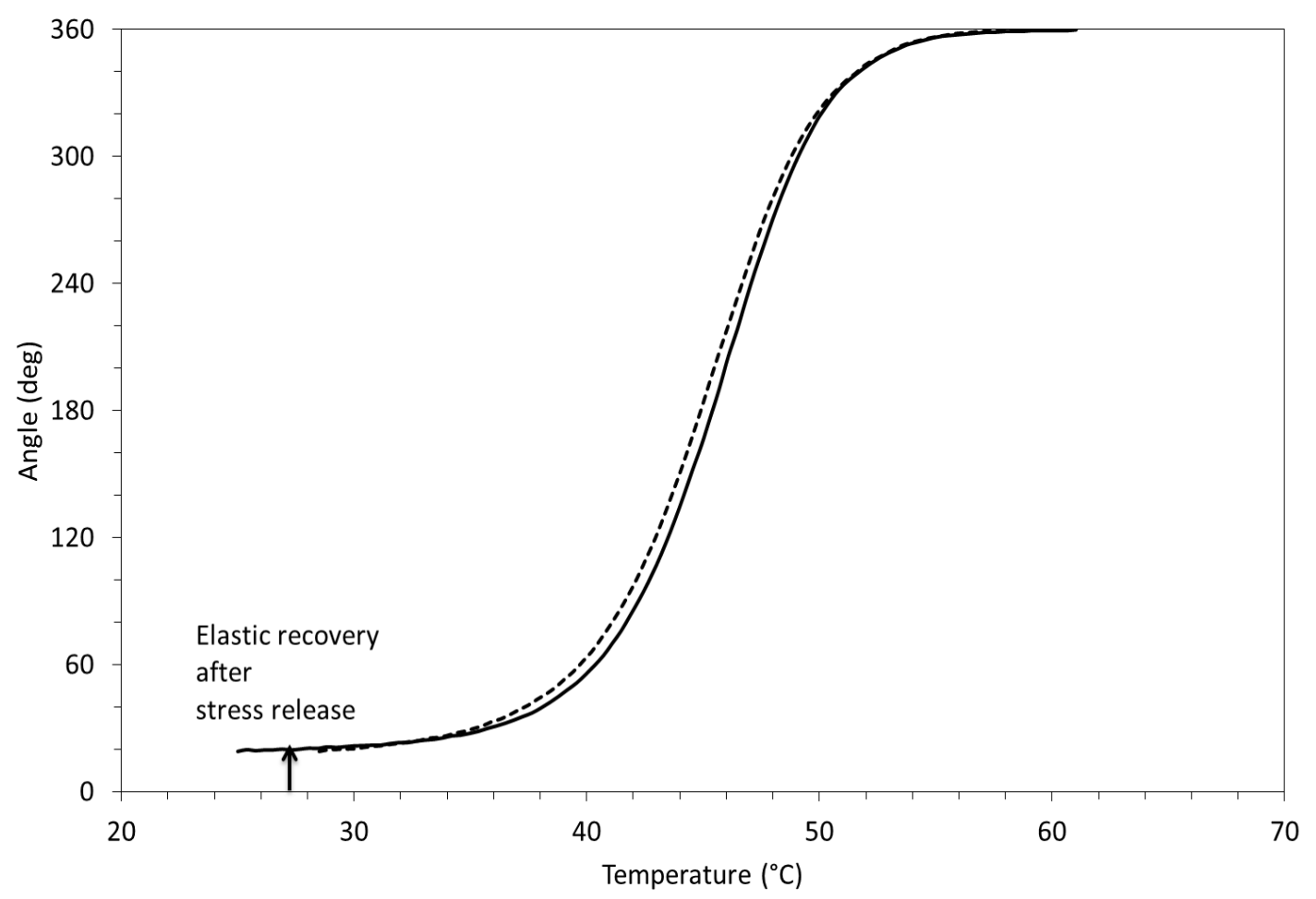

Fig. 6. Torsion angle recovery vs. temperature during stress-free heating at a constant temperature ramp of $0.9^{\circ} \mathrm{C} / \mathrm{min}$ for two shape memory epoxy samples.

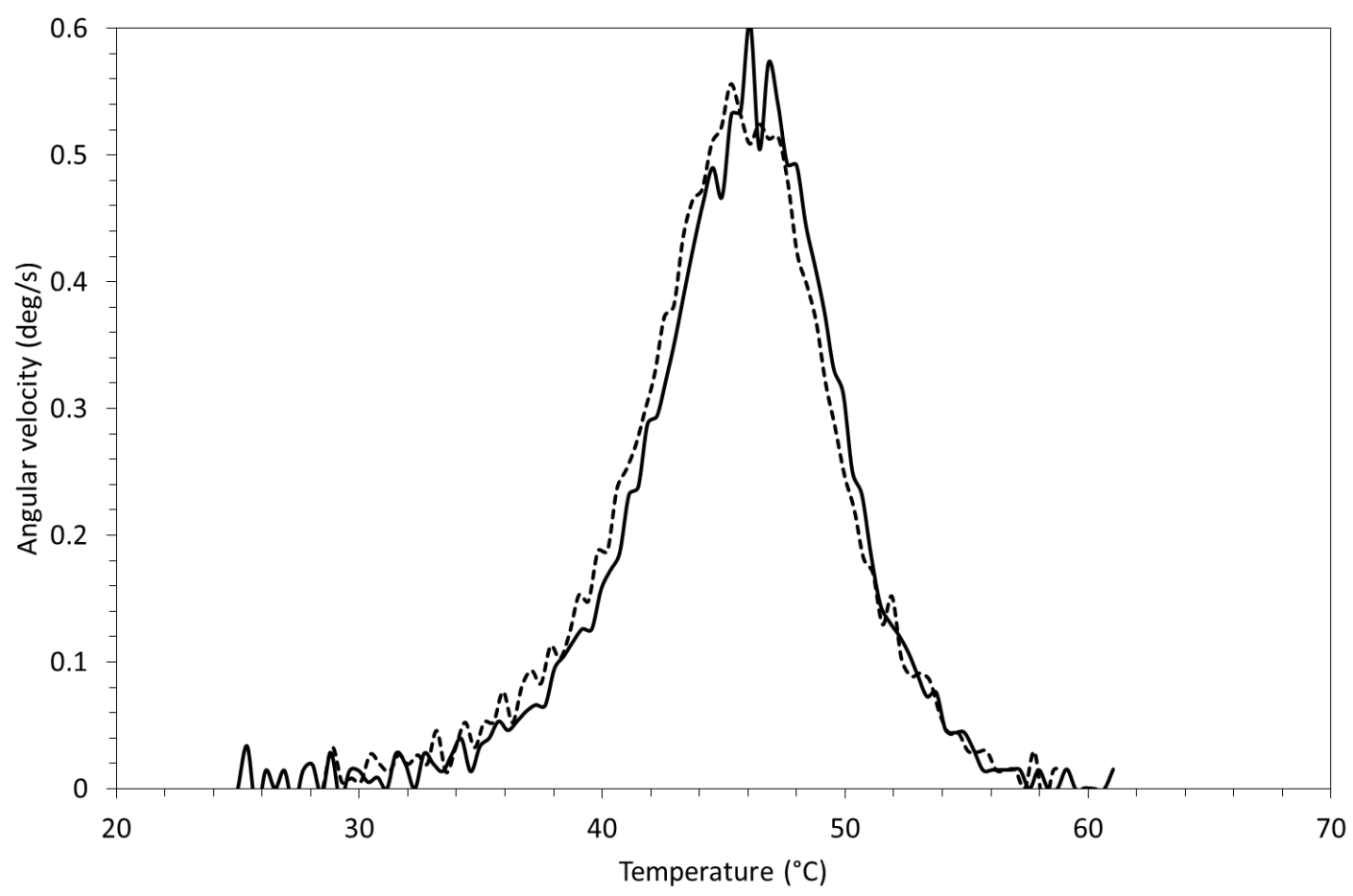

Fig. 7. Angular velocity during torsion recovery vs. temperature during stress-free heating at a constant temperature ramp of $0.9^{\circ} \mathrm{C} / \mathrm{min}$ for two shape memory epoxy samples. 
The interest of the test lies in its ability to provide quantitative measurements of the shape fixity, the shape recovery, and the recovery kinematics and kinetics for large deformations at moderate strains, which are more likely in actual shape memory applications compared to the large deformations at large strains usually characterized by uniaxial tension measurements. In the next section, results are discussed that illustrate the impact of such different test parameters.

\subsection{Effect of the testing parameters}

As designed, a sample may be constrained in a temporary shape characterized by the angle of rotation imposed at the sample's top end. The kinematics of recovery as a function of the applied deformation angle were compared under three different angles of deformation. Fig. 8 shows that the activation temperature of recovery varies with the amount of deformation applied and decreases with increasing deformation. This result may be particularly interesting when studying the macromolecular motions involved during the deformation process at high temperature.

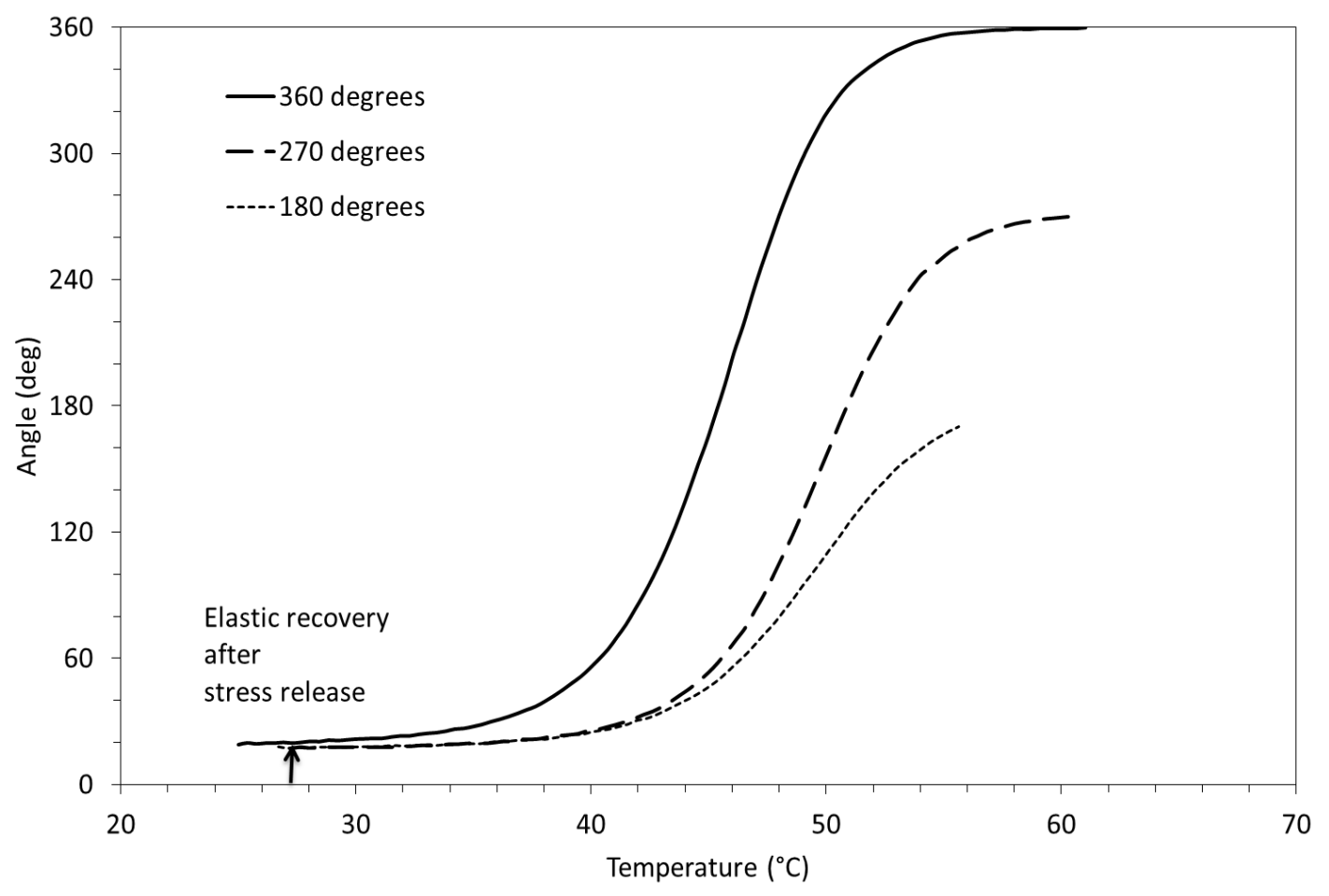

Fig. 8. Kinetics of strain recovery according to the state of deformation of the temporary shape of three epoxy samples submitted to 180,270 and $360^{\circ}$ deformations. Torsion angle recovery versus temperature during stress-free heating at $0.9^{\circ} \mathrm{C} / \mathrm{min}$.

The kinematics of the shape recovery as a function of the heating rate was also investigated. Fig. 9 shows the angular recovery of two epoxy samples previously deformed to 
a full twist (i.e., $360{ }^{\circ}$ deformation angle) when heated at two different heating rates. It was found that a lower heating rate enables the full recovery to be reached at a lower temperature compared to the higher heating rate recovery. This is in good agreement with the results previously reported for similar shape memory epoxies when deformed in tension[22]. Since the recovery is triggered by the activation of molecular mobility, which coincides with a change of viscoelastic properties, this test provides information on the change of viscoelastic properties of a shape memory polymer through its glass, or transformation, transition at large deformation.

In most experimental studies of the shape recovery of SMPs, materials are heated from a low temperature to a high temperature largely exceeding the glass transition temperature. Here, we examined the shape recovery of the epoxy material when exposed to moderately high temperatures. Fig. 10 shows the shape recovery of samples submitted to a full twist and heated at $0.9{ }^{\circ} \mathrm{C} / \mathrm{min}$ up to $42{ }^{\circ} \mathrm{C}$ and $46^{\circ} \mathrm{C}$. As one could expect, the kinematics of recovery differs greatly depending on the ultimate temperature, such that the higher the ultimate temperature, the faster the recovery.

Therefore, beyond SMP testing, the results presented in Fig. 6 through Fig. 10 demonstrate that the proposed torsion device designed and built in this study provides a useful tool for studying the kinetics of molecular mobility through the glass transition of any polymer networks submitted to large, non homogeneous deformations.

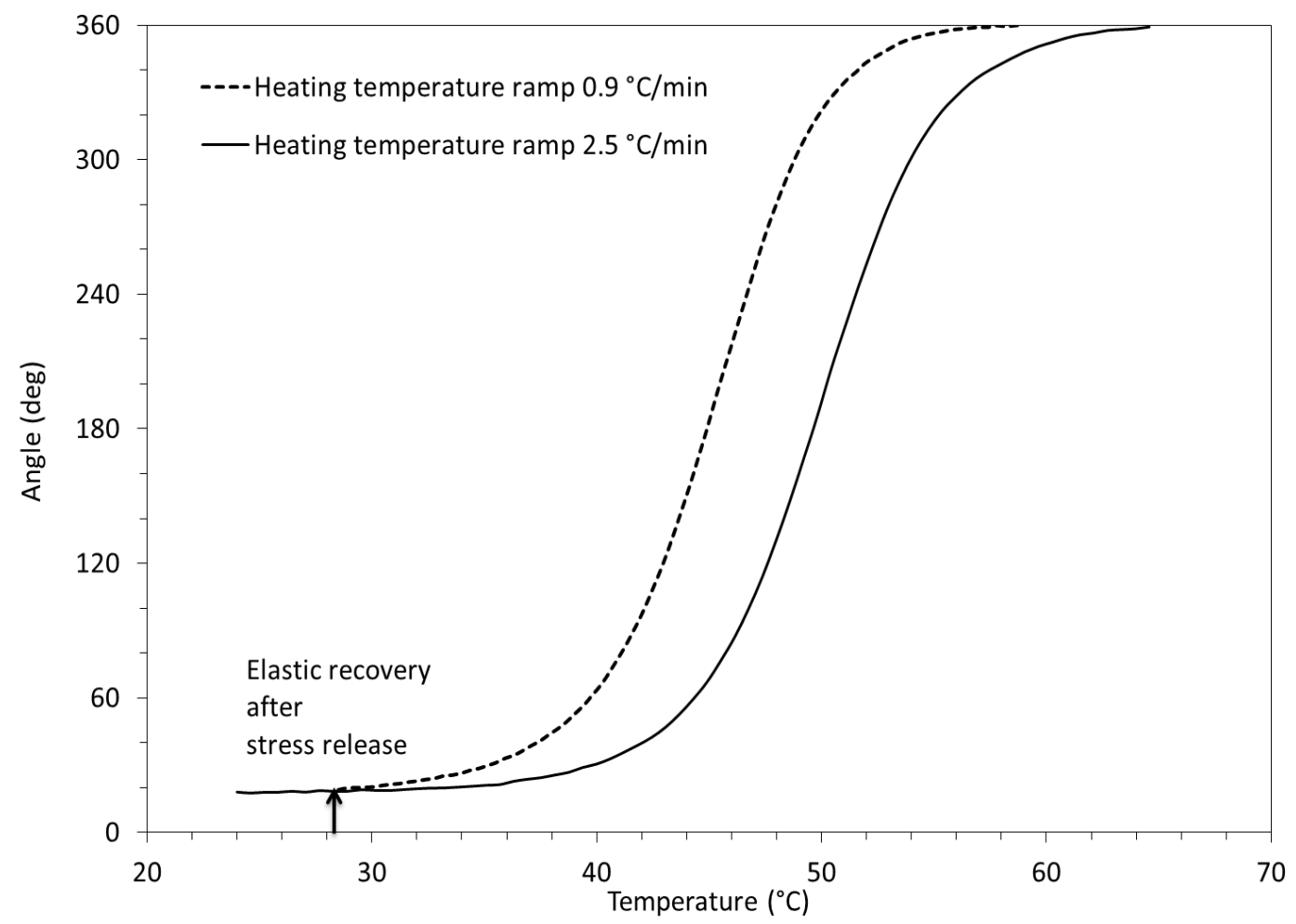

Fig. 9. Angle recovery of a $360^{\circ}$ deformed epoxy sample versus temperature as a function of heating rate. 


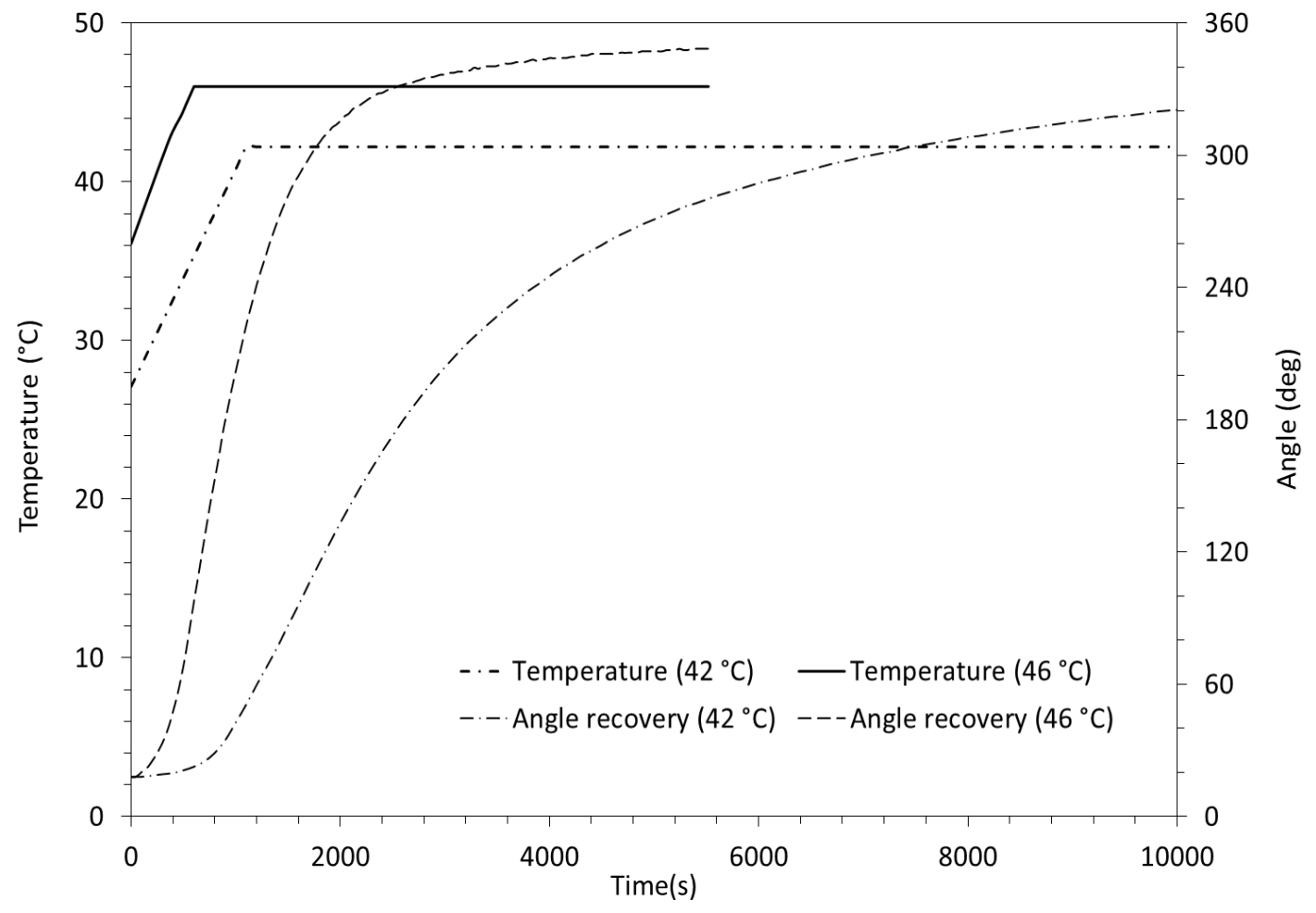

Fig. 10. Angle recovery of a temporarily deformed epoxy sample versus time as a function of the ultimate recovery temperature.

\section{3. $\quad$ Effect of the Materials Composition and Structure}

A major motivation for designing the discussed torsion device was to create a tool that would enable the direct comparison of the shape memory properties of structurally and molecularly different SMPs under large deformations at moderate strains. Indeed, when tested under non-uniaxial loading conditions, the shape memory properties of materials reported in the literature are impossible to compare. However, Fig. 11 clearly illustrate that the torsion test method developed here enables direct comparison of the SM properties of two structurally (i.e., thermoplastic vs. thermoset) and molecularly (i.e., epoxy vs. polyurethane) different SMPs. The thermoplastic polyether block amid (Pebax 7233) was submitted to a $180^{\circ}$ twist at high temperature, and then cooled to room temperature where the deforming stress was released. The shape fixity and shape recovery of the Pebax sample was measured and plotted against those of the epoxy network obtained in a similar manner. Evidently, the elastic recovery, kinematics of recovery, and the amount of recovery differ greatly between the two materials as seen in Fig. 11. The thermoplastic SMP shows a large spontaneous elastic recovery after unloading, synonymous with poor shape fixity. This is rationalized by the relatively high rubbery to glassy modulus ratio (i.e, $\sim 0.1$ for the Pebax and of $\sim 0.01$ for the epoxy). The elastic strain resulting from the stress release is proportional to the applied 
elastic strain at high temperature and the rubbery to glassy modulus ratio, it is therefore significantly larger for the Pebax than for the epoxy. The kinematics of recovery for Pebax 7233 are slow and corroborate its lower storage modulus temperature dependence, as measured by DMA (Fig. 5), compared to the epoxy SMP. Finally, Pebax 7233 exhibits a nonnegligible unrecovered deformation of $11^{\circ}$. This is due to its low elastic strain limit at high temperatures. When applying a $180^{\circ}$ rotation at high temperature, the overall Pebax deformation is the result of both elastic and plastic components.

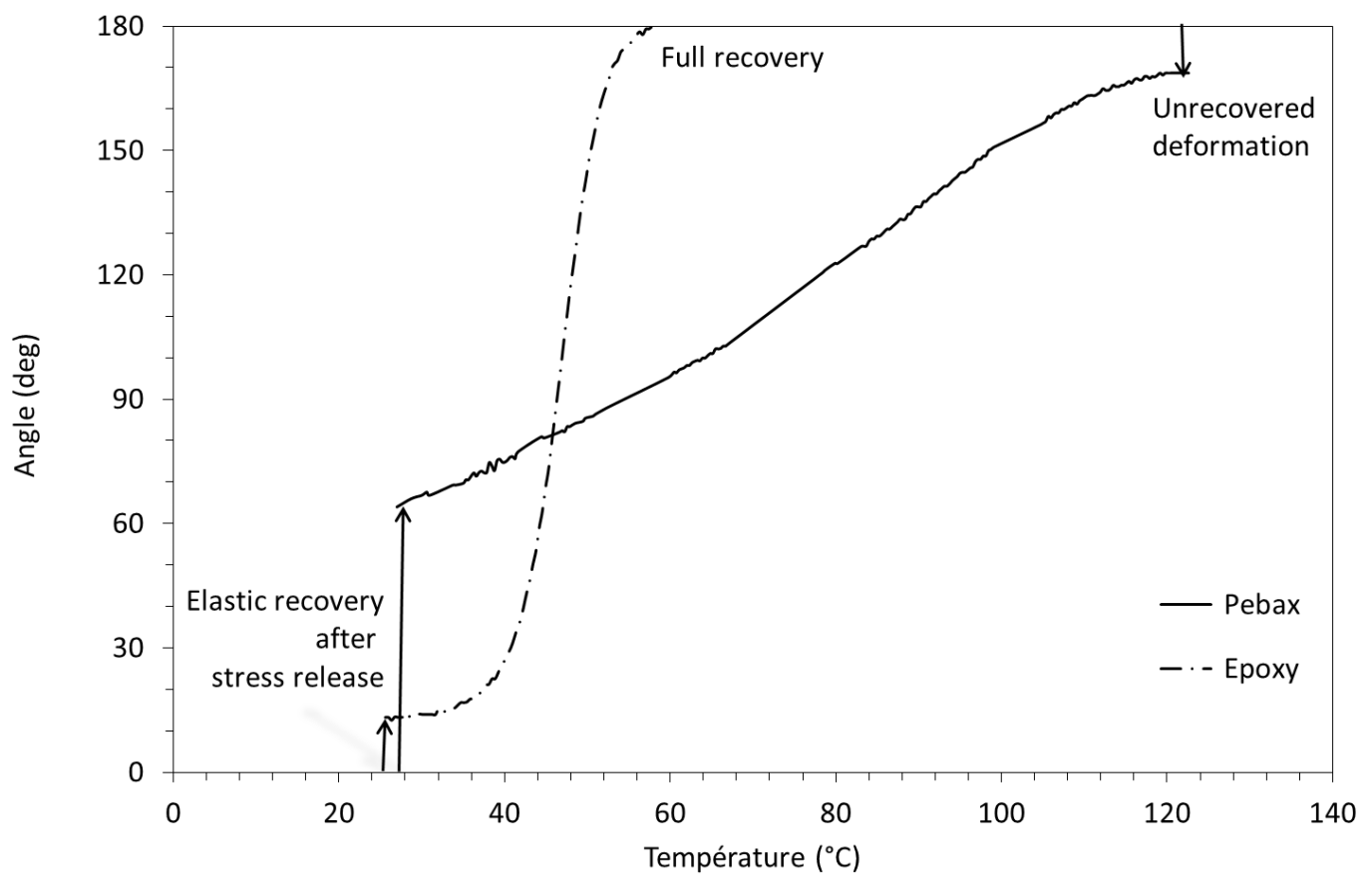

Fig. 11. Torsion angle recovery as a function of temperature for two molecularly and structurally different shape memory polymers during stress-free heating at $0.9{ }^{\circ} \mathrm{C} / \mathrm{min}$.

\section{Conclusion}

An original torsion device was designed and built to enable accurate representation and quantifiable measurements of the shape recovery of SMPs submitted to large deformations at moderate strain levels. Indeed, the states of strains imposed by torsion are believed to represent more accurately those encountered in most end-use applications (i.e., large deformations and moderate strains) compared to those imposed by the traditionally used uniaxial tension or compression deformations (i.e., large deformations and large strains). Moreover, the proposed device is inexpensive and uses only a simple thermal chamber for controlling the testing thermal environment and a CCD camera for monitoring and recording the shape recovery. 
Although torsion is applied instead of the commonly used uniaxial tension or compression, the testing protocol used here follows a similar thermomechanical cycle as that used in traditional shape memory effect testing. The shape/angle recovery is monitored through video capture and quantified by further image analysis using the free ImageJ software. The angle recovery was investigated under varying angle magnitudes, heating rates, and ultimate recovery temperatures. Two SMPs were investigated: a thermoset shape memory epoxy and a thermoplastic shape memory polyurethane (Pebax 7233). Quantitative evaluation of the kinematics and kinetics of the shape recovery of both SMPs was obtained, thereby demonstrating the benefit in using the proposed device and testing protocol described in the report to compare the shape memory properties of structurally and molecularly different materials. This work aims at providing analogous data regardless of materials composition and structure for implementing, fitting and validating generalized SMP thermomechanical models.

\section{Acknowledgement}

Access to the thermal chamber and CCD camera was kindly supplied by Dr. B. Fayolle.

\section{References}

[1] C. Liu, H. Qin, P.T. Mather, Review of progress in shape-memory polymer. J. Mater. Chem. 17 (2007) 1543-1558

[2] G.P. McKnight, C.P. Henry, Large strain variable stiffness composites for shear deformations with applications to morphing aircraft skins, Proceedings of SPIE (2008) 6929, 692919/692911-692919/692912

[3] W.M. Sokolowski, S.C. Tan, Advanced self-deployable structures for space applications.

J. Spacecraft Rockets 44 (2007) 750-54

[4] K. Gall, C.M. Yakacki,Y. Liu, R. Shandas, N. Willett, K.S. Anseth, Thermomechanics of the shape memory effect in polymers for biomedical applications. J. Biomed. Mater. Res. A 73 (2005) 339-348.

[5] C.M. Yakacki, R. Shandas, D. Safranski, A.M. Ortega, K. Sassaman, K. Gall, Strong, tailored, biocompatible shape-memory polymer networks. Adv. Funct. Mater. 18 (2008) 2428-2435.

[6] D.L. Safranski, K. Gall, Effect of chemical structure and crosslinking density on the thermo-mechanical properties and toughness of (meth)acrylate shape memory polymer networks. Polymer 49 (2008) 4446-4455.

[7] T. Xie, I.A. Rousseau, Facile tailoring of thermal transition temperatures of epoxy shape memory polymers. Polymer 50 (2009) 1852-1856.

[8] H. Tobushi, H. Hara, E. Yanmada, S. Hayashi, Thermomechanical properties in a thin 
film of shape memory polymer of polyurethane series . Smart Mater. Structu. 5 (1996) 483491.

[9] H. Tobushi, S. Hayashi, KHoshio, N. Miwa, Influence of strain-holding conditions on shape recovery and secondary-shape forming in polyurethane-shape memory polymer. Smart Mater. Struct. 15 (2006) 1033-1038.

[10]Y. Liu, K. Gall, M.L. Dunn, A.R. Greenberg, J. Diani, Thermomechanics of shape memory polymer: Uniaxial experiments and constitutive modeling. Int. J. Plasticity 22(2) (2006) 279-313.

[11] H.A. Khonakdar, S.H. Jafari, S. Rasouli, J. Morshedian, H. Abedini, Investigation and modeling of temperature dependence recovery behavior of shape-memory crosslinked polyethylene. Macromol. Theory Simul. 16 (2007) 43-52.

[12] K. Gall, M.L. Dunn, Y. Liu, Internal stress storage in shape memory polymer nanocomposites. Appl. Phys. Letters 85(1) (2004) 1-3.

[13] H.J. Qi, T.D. Nguyen, F. Castro, C.M. Yakacki, R. Shandas, Finite deformation thermomechanical behavior of thermally induced shape memory polymers. J. Mech. Phys. Solids 56 (2008) 1730-1731.

[14] K.K. Westbrook, F. Castro, K.N. Long, A.J. Slifka, H.J. Qi, Improved testing system for thermomechanical experiments on polymers using uniaxial compression equipment. Polymer Testing 29 (2010) 5013-512.

[15] M. Behl, A. Lendlein, Shape memory polymers. Materials today 10(4) (2007) 20-28.

[16] J.R. Lin, L.W. Chen, Shape-memorized crosslinked ester-type polyurethane and its mechanical viscoelastic model. J. Appl. Polym. Sci. 73 (1999) 1305-1319.

[17] K. Gall, M.L. Dunn, Y. Liu, D. Finch, M. Lake, N.A. Munshi, Shape memory polymer nanocomposites. Acta Mater. 50 (2002) 5115-5126.

[18] T. Xie, X. Xiao, Y.T. Cheng, Revealing triple -shape memory effect by polymer bilayers. Macromol. Rapid Commun. 30 (2009) 1823-1827.

[19] A. Lendlein, A.M. Schmidt, R. Langer, AB-polymer networks based on oligo( $\varepsilon-$ caprolactone) segments showing shape-memory properties. Proc. Natl. Acad. Sci. USA 98(3) (2001) 842-847.

[20] Rasband, W.S., ImageJ, U. S. National Institutes of Health, Bethesda, Maryland, USA, http://rsb.info.nih.gov/ij/, 1997-2009.

[21] I. F. Sbalzarini, P. Koumoutsakos, Feature point tracking and trajectory analysis for video imaging in cell biology. J. Struct. Biol. 151(2) (2005) 182-195.

[22] I.A. Rousseau, T. Xie, Shape memory epoxy: composition, structure, properties and shape memory performances. J. Mater. Chem. 20 (2010) 3431-3441. 